\title{
Molecular dynamics simulation of aluminium melting
}

\section{Simulacija taljenja aluminija z uporabo metode molekularne dinamike}

\author{
Jakob Novak \\ Faculty of Mathematics and Physics, University of Ljubljana, Jadranska ul. 19, 1000 Ljubljana, Slovenia \\ novak.jakob@yahoo.com
}

\begin{abstract}
Solid-liquid phase transition has been simulated by the molecular dynamics method, using isobaric-isoenthalpic ensemble. For interatomic potential, glue potential has been selected. The original algorithm for bookkeeping of the information on neighbouring relationships of the atoms has been developed and used in this research. Time consumption for calculation of interatomic forces has been reduced from $o\left(N^{2}\right)$ to $o(N)$ by the use of this algorithm.

Calculations show that phase transition from solid to liquid occurs between 1,000 $\mathrm{K}$ and 1,300 K. The simulated temperature of phase transition is higher than the experimental value due to the absence of crystal defects. If constant heat flux is supplied, temperature decreases during melting because the superheated state becomes unstable. During the cooling process, no significant changes of the observed variables were detected due to the high cooling rate, which prevents crystallisation.
\end{abstract}

Key words: molecular dynamics simulation, isobaricisoenthalpic ensemble, glue potential, bulk aluminium melting

\section{Izvleček}

Fazni prehod trdno-tekoče je bil simuliran z metodo molekularne dinamike, z uporabo izobarnega-izoentalpnega ansambla. Za medatomski potencial je bil uporabljen večdelčni potencial, ki ga je razvil F. Ercolessi (ang. Glue potential) [1]. Originalen algoritem, za shranjevanje informacije o sosedskih odnosih med atomi, je bil razvit in uporabljen v pričujoči raziskavi. Časovna poraba izračuna medatomskih sil je z njegovo pomočjo zmanjšana iz $o\left(N^{2}\right)$ na $o(N)$.

Izračuni kažejo, da dobimo fazni prehod iz trdnega $v$ tekoče, med 1000 K in 1300 K. Simulirana temperatura faznega prehoda je višja od eksperimentalne vrednosti, zaradi odsotnosti defektov v kristalu. Če dovajamo konstanten toplotni tok, med taljenjem temperatura vzorca pade, saj postane pregreto stanje nestabilno. Pri ohlajanju taline, ni bilo zaznanih signifikantnih sprememb v opazovanih spremenljivkah, zaradi visokih hitrosti ohlajanja, ki preprečijo kristalizacijo.

Ključne besede: metoda molekularne dinamike, izobaren-izoentalpen ansambel, večdelčni medatomski potencial, taljenje razsežnega aluminija 


\section{Introduction}

Melting and solidification constitute an important segment of metal alloy production. The process of phase transition is influenced by many parameters. One of them is the interatomic potential energy, which is the consequence of previous processing of the material. This study is an introduction to research into the influence of crystal defects on the characteristics of solid-liquid phase transition of metals. Our special point of interest is the influence of defects on the enthalpy of fusion and short-range order in the melt.

To observe the influence of crystal defects on the behaviour of a sample, simulation, at the atomic level, is required. Two different methods are commonly used for simulating the behaviour of materials, on the basis of their atomic configuration: molecular dynamics and Monte Carlo simulation [2]. Both methods extract macroscopic quantities from the time evolution of particles on the microscopic level. The difference is that in molecular dynamics, time evolution is deterministic, in contrast to Monte Carlo simulations, wherein atomic states are chosen randomly for every time step, according to suitable distributions. An example of kinetic Monte Carlo simulation considering asymmetric diffusion in $\mathrm{Fe}-\mathrm{Cu}$ alloy is presented in the study by Bombač and Kugler [3]. In this research, the molecular dynamics method has been chosen, because it automatically includes the influence of sample history on the behaviour of the material, which is much harder to incorporate in Monte Carlo simulations. This is very important for the study of the influence of crystal defects on the phase transition.

In literature, molecular dynamics simulations are used for the examination of different processes in materials, e.g., for calculation of transport and equilibrium diffusivity in LennardJones fluids [4] and for investigation of surface diffusion [5]. Molecular dynamics simulation has also been widely used for examination of the behaviour of crystal defects. In previous studies [6,7], migration of grain boundaries has been simulated; some studies [8,9] discuss the dislocation processes as a consequence of mechanical deformation in aluminium.
In this study, simulation of aluminium phase transition is presented. Crystal defects have not been applied yet. The sample is an ideal, infinite, face-centred cubic (FCC) crystal. The infinite size of the crystal has been accomplished by periodic boundary conditions. The reason for application of periodic boundary conditions is that the current computational capabilities typically enable molecular dynamics simulations of samples of sizes up to $10 \mathrm{~nm}$. This type of size is satisfactory for simulating the behaviour of nanoparticles, but it does not satisfy for observation of macroscopic sample, so called bulk aluminium. Even though we are dealing with the same element in the case of nanoparticle and bulk aluminium, there are important differences in the properties of the two samples [10]. The latter has no free surface. This results in different physical properties of phase transition because the free surface acts as a nucleation site for phase transition [11].

\section{Theoretical introduction}

In this study, the isobaric-isoenthalpic ensemble (NPH) has been selected because it allows energy and volume to fluctuate. This is not the case in a microcanonical ensemble (NVE), which uses Newtonian equations of motion and periodic boundary conditions. Energy and volume fluctuations are very important for the description of phase transition because energy fluctuations and thermal expansion affect the melting point. The isobaric-isoenthalpic ensemble uses volume as the additional degree of freedom to describe the coupling of the observed sample with its surroundings. The equations of motion for $N$ atoms are expressed as follows [12]:

$M \ddot{V}=-P+\frac{1}{3 V}\left(V^{\frac{2}{3}} \sum_{i} m_{i}\left|\dot{\vec{\rho}}_{i}\right|^{2}+V^{\frac{1}{3}} \sum_{i<j} \vec{F}_{i j} \cdot \vec{\rho}_{i j}\right)$

and

$m_{i} \ddot{\vec{\rho}}_{i}=V^{-\frac{1}{3}} \sum_{j \neq i} \vec{F}_{i j}-2 m_{i} \dot{V}^{\dot{\rho}_{i}} / 3 V$

where $M$ denotes a constant fictitious mass, $m_{\mathrm{i}}$ is the mass of the atom $i, V$ is the volume of the 

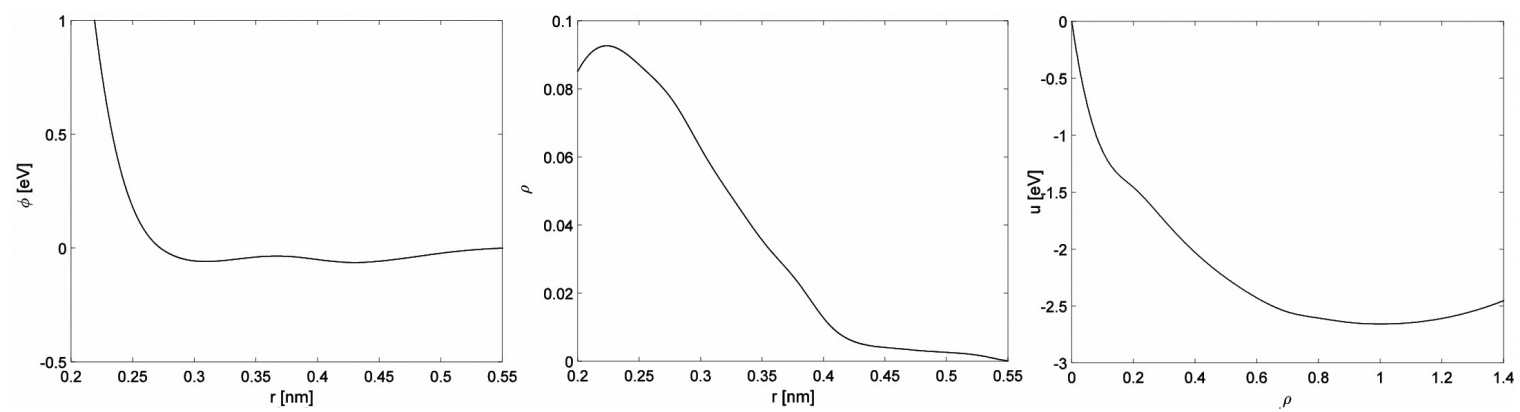

Figure 1: Calculated glue potential for aluminium [1].

sample (treated as a dynamic variable), $P$ is the pressure, $\vec{\rho}_{i}$ is the scaled position of atom $i, \vec{\rho}_{i j}$ is the distance from atom $i$ to the atom $j$ in the scaled coordinate system and $\vec{F}_{i j}$ is the force of atom $i$ to the atom $j$. The fictitious mass $M$ only affects the time scale of the volume fluctuations and does not affect the time averages of physical quantities. Its value should be chosen so that the time scale of the volume fluctuations is approximately equal to $V^{1 / 3} / c$, where $c$ denotes the speed of sound in the simulated material [12]. In this research, its value has been set as $\mathrm{M}=610 \mathrm{keV} / \mathrm{c}^{2}$.

The macroscopic thermodynamic properties of the system are derived by averaging the instantaneous values over time. For example, the average of some physical quantity can be evaluated as follows [11]:

$$
\langle A\rangle=\frac{1}{N_{\text {step }}} \sum_{i=1}^{N_{\text {step }}} A\left(\vec{R}_{i}, \overrightarrow{\mathrm{V}}_{i}\right) \text {, }
$$

where $\vec{R}_{i}=\left(\vec{r}_{1}, \ldots, \vec{r}_{N}\right)$ and $\overrightarrow{\mathrm{V}}=\left(\vec{v}_{1}, \ldots, \vec{v}_{N}\right)$ denote the vectors of coordinates and velocities of all $N$ particles in the sample, which are related to the scaled coordinates by the following relations: $\vec{r}_{i}=V^{1 / 3} \vec{\rho}_{i}$ and $\vec{v}_{i}=V^{1 / 3} \dot{\vec{\rho}}_{i} . N_{\text {step }}$ denotes the number of steps taken into account in the average. The results presented in this study are chosen to be averaged over 5,000 time steps. Application of Eq. (3) gives the expression for temperature:

$T=\frac{\left\langle\sum_{i} m_{i} v_{i}^{2}\right\rangle}{3 N k_{B}}$

Another quantity used in this study is the translational order parameter [11]:
$\lambda=\frac{1}{N} \sqrt{\sum_{i=1}^{N} \cos \left(\vec{k} \cdot \vec{r}_{i}\right)+\sum_{i=1}^{N} \sin \left(\vec{k} \cdot \vec{r}_{i}\right)}$,

$\vec{k}=\frac{\left((2 N)^{\frac{1}{3}} \pi\right)}{D}(-1,1,1)$,

where $\vec{k}$ is an arbitrary vector of the reciprocal lattice and $D$ is the side length of the simulation domain. The translational order parameter is a measure of the structure present in the solid. It can take values between zero and one, where one means an ideal crystal and zero indicates the total absence of order.

The glue form of the potential has been used to evaluate the interatomic forces [1]:

$U_{\text {glue }}\left(r_{i j}\right)=\frac{1}{2} \sum_{i, j} \varphi\left(r_{i j}\right)+\sum_{i} u\left(\sum_{j} \rho\left(r_{i j}\right)\right)$

Functions $\varphi\left(r_{i j}\right), \rho\left(r_{i j}\right)$ and $u(\rho)$ are extracted numerically from the large amounts of data produced, by the first-principles calculations, whereby forces on atoms are obtained by solving the electronic structure problem using quantum mechanics. The three functions constituting the glue potential were calculated by the force-matching method and are presented in Figure 1. This method compares the forces, computed by the first principles for many different configurations of atoms to those produced by the molecular dynamics simulation using the test potential. The test potential has a set of $L$ parameters: $\vec{p}=\left(p_{1}, \ldots, p_{L}\right)$, the values of which have to be adjusted such that the best agreement with the first-principles calculations is achieved. This is accomplished by minimising the objective function $Z(\vec{p})$ as follows [1]: 
$Z(\vec{p})=Z_{F}(\vec{p})+Z_{C}(\vec{p})$,

$Z_{F}(\vec{p})=\left(3 \sum_{k=1}^{K} N_{k}\right)^{-1} \sum_{k=1}^{K} \sum_{i=1}^{N_{k}}\left|\vec{F}_{k i}(\vec{p})-\vec{F}_{k i}^{0}\right|^{2}$

$Z_{C}(\vec{p})=\sum_{r=1}^{N_{c}} W_{r}\left(A_{r}(\vec{p})-A_{r}^{0}\right)^{2}$.

In $Z_{F^{\prime}} K$ is the number of sets of atomic configurations available, $N_{K}$ is the number of atoms in configuration $k, \vec{F}_{k i}(\vec{p})$ is the force on the atom $i$ in set $k$, as obtained with parametrisation $\vec{p}$, and $\vec{F}_{k i}^{0}$ is the reference force from first principles. $Z_{C}$ contains contributions from $N_{c}$ additional constraints. $A_{r}(\vec{p})$ represents some selected physical quantities as calculated with parametrisation $\vec{p}$. $A_{r}^{0}$ stands for the corresponding reference quantities, which may be supplied either from the first-principles calculation or directly from experiments. $W_{r}$ represents the weights that are chosen at convenience [1].

In the simulation, the initial positions and the velocities of atoms are chosen randomly, according to Maxwell-Boltzmann distribution. The probability of atoms to be located at positions $\vec{R}_{i}=\left(\vec{r}_{1}, \ldots, \vec{r}_{N}\right)$ and having velocities $\overrightarrow{\mathrm{V}}=\left(\vec{v}_{1}, \ldots, \vec{v}_{N}\right)$ is expressed as follows:

$d w=A \exp \left(-\left(U(\vec{R})+\sum_{i} \frac{1}{2} m_{i} v_{i}^{2}\right) / k_{B} T\right) d \vec{R} d \overrightarrow{\mathrm{V}}$

where $k_{B}$ is the Boltzmann constant, $T$ is the temperature, $m_{i}$ is the mass of particle $i, U$ is the interatomic potential and $A$ is the normalisation constant. As long as the sample is in the solid phase, vector $\vec{R}$ can only contain values near the global minimum of potential energy, $\vec{R}_{0}$, which would be, as we know from experiment, an FCC lattice with lattice parameter $a=$ $0.4046 \mathrm{~nm}$ [13] for aluminium. Vector $\vec{R}_{0}$ is calculated using a cubic Bravais lattice, with basis. Vectors of the Bravais lattice are as follows:

$\vec{r}_{B}\left(n_{1}, n_{2}, n_{3}\right)=n_{1} \vec{a}_{1}+n_{2} \vec{a}_{2}+n_{3} \vec{a}_{3}$,

where $\quad \vec{a}_{1}=(a, 0,0), \quad \vec{a}_{2}=(0, a, 0) \quad$ and $\vec{a}_{3}=(0,0, a)$ are primitive vectors of the cubic Bravais lattice and $n_{1}, n_{2}$ and $n_{3}$ can be any integer. For the FCC lattice, at every point of the Bravais lattice, a basis consisting of four atoms is placed. The positions of atoms are therefore determined by the Bravais lattice vector and one of the four basis vectors: $\vec{r}_{0}\left(n_{1}, n_{2}, n_{3}, k\right)=\vec{r}_{B}\left(n_{1}, n_{2}, n_{3}\right)+\vec{b}_{k}$

$k=1,2,3,4$

$\vec{b}_{1}=(0,0,0)$,

$\vec{b}_{2}=\frac{1}{2}(a, a, 0)$,

$\vec{b}_{3}=\frac{1}{2}(a, 0, a)$,

$\vec{b}_{4}=\frac{1}{2}(0, a, a)$.

Vector $\vec{R}$ can be expressed as small fluctuations $\overrightarrow{\mathrm{E}}=\left(\vec{\epsilon}_{1}, \ldots, \vec{\epsilon}_{N}\right)$ around the minimum: $\vec{R}=\vec{R}_{0}+\vec{E}$. After the Taylor expansion, Eq. (11) gets the following form:

$$
\begin{aligned}
d w= & A^{\prime} \exp \left(-\left(\sum_{i, j} \frac{1}{2} \vec{\epsilon}_{i} \cdot \frac{\partial^{2} U}{\partial \vec{r}_{i} \partial \vec{r}_{j}}\left(\vec{R}_{0}\right) \vec{\epsilon}_{j}+\right.\right. \\
& \left.\left.+\sum_{i} \frac{1}{2} m_{i} v_{i}^{2}\right) / k_{B} T\right) d \overrightarrow{\mathrm{E}} d \overrightarrow{\mathcal{V}}
\end{aligned}
$$

Velocities of particles are the independent degrees of freedom because the probability for a particle to have certain velocity is independent of the velocities and positions of other particles. But that is not the case for positions. For that reason, transition to normal coordinates, $\vec{q}_{i}$, is necessary. Normal coordinates are the normal modes of the crystal. They are obtained by diagonalisation of the potential $U$. Amplitude of the normal mode, $q_{i}=\left|\vec{q}_{i}\right|$, becomes the independent degree of freedom and probability for it can be chosen randomly, according to probability distribution [14]:

$d w\left(q_{i}\right)=A^{\prime \prime} \exp \left(-m_{i} \omega_{i}^{2} q_{i}^{2} / 2 k_{B} T\right) d q_{i}$,

where $\omega_{i}$ is the eigenfrequency of the crystal, calculated from the eigenvalues of the matrix of second derivatives of the potential energy. Velocities can be determined in the standard coordinates

$d w\left(v_{i}\right)=A^{\prime \prime \prime} \exp \left(-m_{i} v_{i}^{2} / 2 k_{B} T\right) d v_{i}$.

Eqs. (15) and (16) are useful only if we know the minimum vector $\vec{R}_{0}$. In the case of ideal FCC lattice, we know the global minimum from experiments; however, if crystal defects are present in the solid, it is much harder to determine the exact vector of the local minimum. That is why this research has been carried out to investigate how fast atoms equilibrate. The conclusion is that there is no need to set correct initial conditions, but an arbitrary initial condition can be selected, after which atoms spontaneously transit into the equilibrium state on 
a time scale shorter in comparison to the simulation running time.

\section{Computational framework}

All the results presented in this study were computed with the code written especially for the needs of this research, and no commercial program for molecular dynamics has been used. In this section, the algorithms constituting the simulation are presented, with emphasis on the algorithm for bookkeeping of neighbouring relationships of the atoms, which has been developed for the needs of this study and differs from the commonly used Verlet list or Linked list.

The sample observed was a cube, with initial side length of $3.24 \mathrm{~nm}$, consisting of 2,048 atoms. Vectors of Bravais lattice were parallel to the cube sides. To simulate the melting of bulk aluminium, periodic boundary conditions were applied. When an atom passes through the face of a cube, it enters through the opposite face of the cube. Atoms located at the surface of the cube also feel the potential of the atoms from the opposite side. With this procedure, an effectively infinite crystal is generated.

The system of second-order differential equations (1) and (2) has been solved numerically by the Gear predictor corrector algorithm [15]. This algorithm is especially appropriate for systems with large numbers of particles because it is not time consuming and is a very good energy conserver. The time step has been chosen to be $1 \mathrm{fs}$, considering that the time scale for vibration of atoms is of the same order [11]. During the heating process, constant heat flux is supplied to the sample. At every time step, the average energy per particle is increased by $2.6 \times 10^{-6} \mathrm{eV}$, by scaling the velocities. The same heat flux, with a negative sign, is set for the cooling process. Table 1 shows the list of all parameters used to obtain the results presented in this paper.

The most time-consuming part of the simulation is the computation of forces because the total force on the selected particle is the sum of forces due to all the particles in the simulation. In order to shorten the computational time, only the forces due to the surrounding atoms
Table 1: List of all parameters used in the simulation, with corresponding values.

\begin{tabular}{ll} 
Parameter name & $\begin{array}{l}\text { Parameter } \\
\text { value }\end{array}$ \\
\hline Number of atoms $(N)$ & 2,048 \\
\hline Aluminium lattice parameter $(a)$ & $0.4046 \mathrm{~nm}$ \\
\hline Mass of aluminium atom $(m)$ & $25.15 \mathrm{GeV} / \mathrm{c}^{2}$ \\
\hline Fictitious mass $(M)$ & $610 \mathrm{keV} / \mathrm{c}^{2}$ \\
\hline Integration time step & $1 \mathrm{fs}$ \\
\hline Potential cutoff distance $r_{c}$ & $0.555 \mathrm{~nm}$ \\
\hline Pressure $(P)$ & $0 \mathrm{~Pa}$ \\
\hline Heat flux & $2.6 \times 10^{-6} \mathrm{eV} / \mathrm{fs}$ \\
\hline
\end{tabular}

are calculated; other forces are assumed to be negligible. To reduce time consumption from $o\left(N^{2}\right)$ to $o(N)$, the algorithm that retains the information of neighbouring relationships has been applied, to avoid calculation of the distances due to all atoms. In literature, the most commonly used algorithms for the bookkeeping of neighbouring relationships are the Verlet list and the Linked list. The first one reduces the time consumption to $o\left(N^{3 / 2}\right)[2]$ and is the most efficient for smaller samples. The second reduces the time consumption to $o(N)$ [2], but it is less efficient than the algorithm presented in this study for most of the situations.

The basic principle of the algorithm is the following. Before the simulation starts, every atom is given a unique number, which serves as its identity. Two lists are constructed for every atom. The first one contains the numbers of all atoms situated in a sphere of radius $r_{c}$ (herein referred to as 'near neighbours'), where $r_{c}$ is an arbitrary selected value that represents the range of interatomic interaction. Because we are dealing with a numerically computed potential, $r_{c}$ coincides with the potential cutoff distance. In this study, the cutoff has been set to $0.555 \mathrm{~nm}$ because at this distance, the functions $\varphi\left(r_{i j}\right)$ and $\rho\left(r_{i j}\right)$ become zero to the relative accuracy of $10^{-6}$. The second list contains all the near neighbours of the earlier near neighbours (herein referred to as 'far neighbours'). At every time step, the algorithm checks whether all the near neighbours from the previous time step still lie inside the sphere of radius $r_{c}$. If not, they are moved to the list of far neighbours or, eventually, in a very special case, when they do 


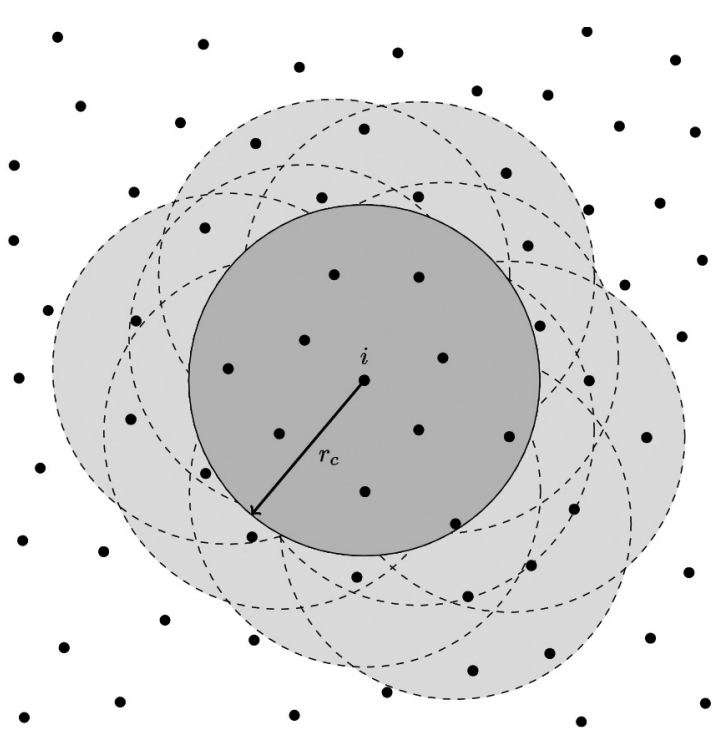

Figure 2: Graphical presentation of the two lists used by the algorithm. The area shaded in a dark grey colour represents the sphere with radius $r_{c}$, which is the potential cutoff radius. Atoms located in this area are the near neighbours of atom $i$. In the light grey is the shaded area, where the far neighbours can be found.

not share any near neighbour with the atom of our interest, they are just deleted from the list of near neighbours. An analogous check is conducted at every time step for the list of far neighbours. Every time a change occurs in any of the two lists of any atom, the lists of all other atoms affected are corrected. The principle of the near- and far-neighbour list is graphically presented in Figure 2. All the main parts of the simulation, described in this section, are gathered and graphically presented in Figure 3.

The herein-presented algorithm for bookkeeping of neighbouring relationships of the atoms assumes that only far neighbours can become near neighbours of the atom in the next time step. This assumption is legitimate in systems with high density because they have very low probability for events in which the atom enters the neighbouring region of the other atom without sharing a common neighbour. This assumption is valid in most of the solid and liquid systems. But it is not valid in the case of a gas.

The time consumption, which depends on the number of atoms that must be taken into account by the calculation of forces, is proportional to the volume of the area where the near or far neighbours can be located. This area is certainly smaller than the sphere of radius $2 r_{c}$. This means that the time consumption of

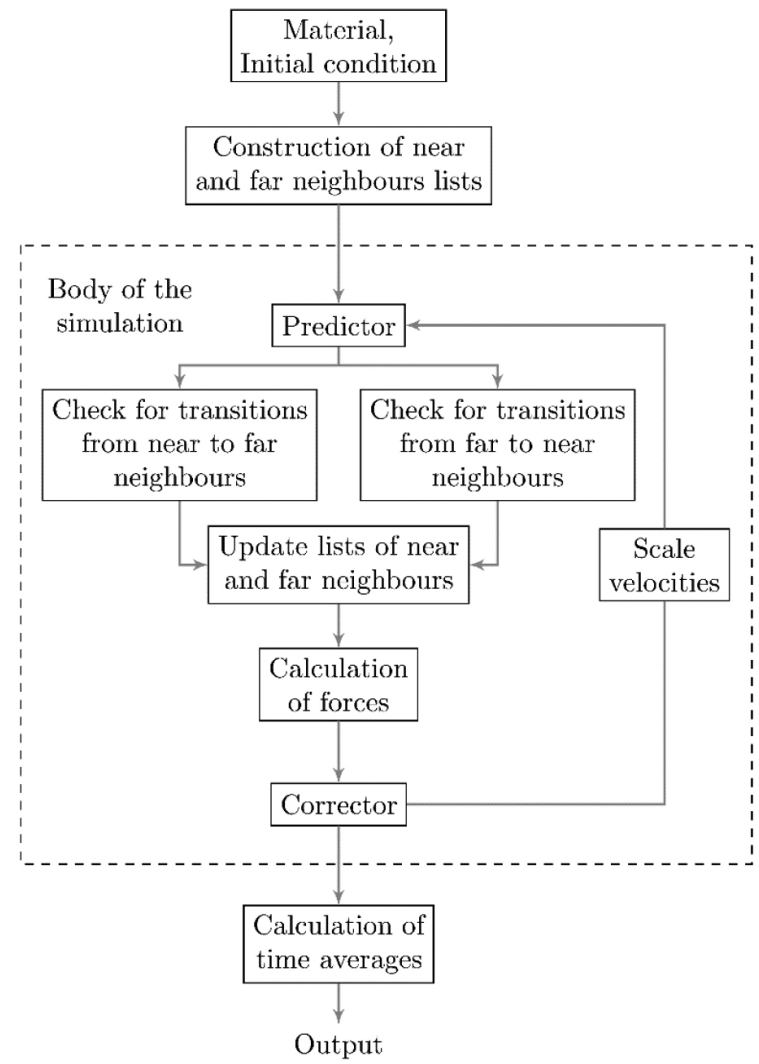

Figure 3: Schematic demonstration of the simulation. The main part of the simulation is integration of equations of motion, with addition of scaling velocities at every time step. Integration has two steps. The first is prediction of the atomic positions and velocities, based on the scaled positions and volume derivatives. The second step is the correction of the predicted positions and velocities, by calculating the interatomic forces at the positions supplied by the predictor [15].

the algorithm is, in the worst case, proportional to $n_{f a r}=32 \pi \rho r_{C}^{3} / 3$. The time consumption of the Linked list algorithm is proportional to $n_{l}=27 \rho r_{C}^{3}[2]$. The ratio of the time consumptions therefore equals, at the most, $n_{f a r} / n_{l}=1.26$. In this case, the Linked list algorithm is a better choice. But this case is very unlikely to happen. In most of the situations, the far neighbours list is more efficient than the Linked list. For example, in the case of an ideal FCC lattice, with aluminium lattice parameter and the range of interaction $r_{c}=0.555 \mathrm{~nm}$, as used in this study, the ratio $n_{f a r} / n_{l}=0.68$. And this ratio can be significantly improved, if the density of the system is high enough, to introduce another radius $r_{f a r}<r_{C}$, which is only used for construction of the list of far neighbours and can significantly decrease the vol- 


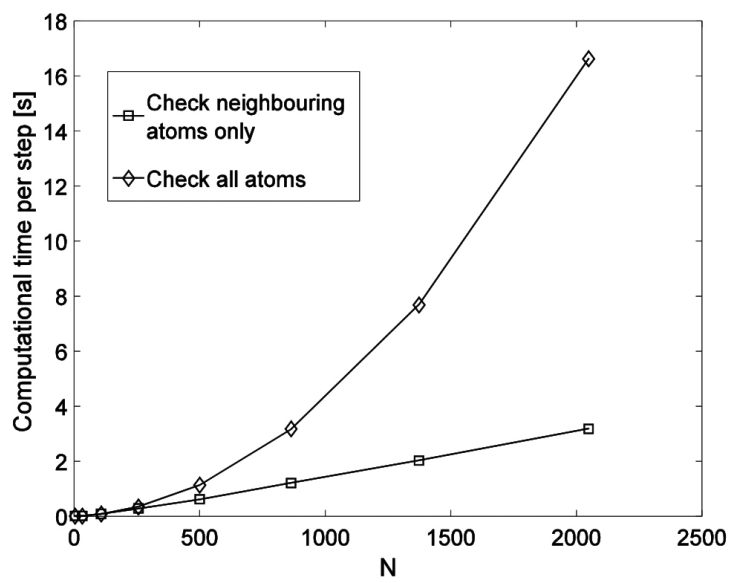

Figure 4: Comparison of time consumption of the standard method for calculating forces, where interacting atoms are determined at every time step, with the method whereby lists of neighbours are held and updated through the simulation run.

ume of the region of far neighbours. The cutoff radius, which determines the list of near neighbours of course remains the same in that case.

\section{Results and discussion}

First, a comparison of the algorithm used in this study relative to the basic method, where distances due to all atoms in the sample are calculated at every time step, is reported. Figure 4 compares the time consumption of the standard method with the method presented in this study. Computational time is measured for the cube consisting of different numbers of atoms, without periodic boundary conditions, at the temperature $300 \mathrm{~K}$. In the case of the sample with 2,048 atoms, simulation runs 5-6 times faster with application of the described algorithm. The difference should be even more significant at higher numbers of atoms in the sample and in the case of periodic boundary conditions because information about the neighbouring relationships of atoms from the opposite sides of a cube can be very simply incorporated in the lists of far and near neighbours.

Before observance of aluminium melting, the influence of the initial condition on the behaviour of the sample during the simulation run has been examined. For the initial condition, an ideal FCC lattice with correct lattice parameter has been set. Velocities were distributed

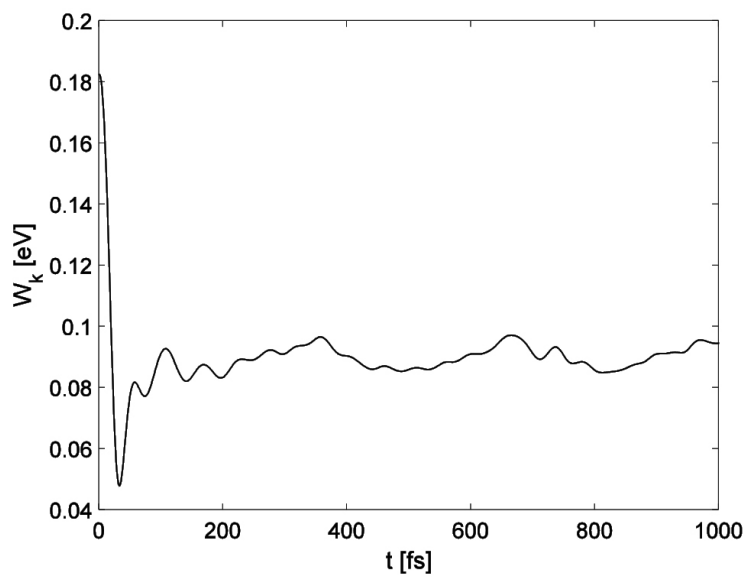

Figure 5: Time dependence of average particle kinetic energy during the first 1,000 time steps.

according to Eq. (16) for an arbitrary selected temperature. As seen in Figure 5, thermodynamic equilibrium is established on a time scale of $0.1 \mathrm{ps}$, when the kinetic energy begins to fluctuate around the fixed value. Half of the initial kinetic energy is transformed to potential energy. This is because the average kinetic energy equals the average potential energy in the case of a harmonic oscillator. Low-frequency oscillations $\left(v \sim 3 p s^{-1}\right)$ are the consequences of volume fluctuations.

The distributions of distances of particles from the corresponding positions in an ideal FCC lattice, as well as the velocity distribution among the particles after running the simulation for 1 ps were compared with those calculated from Eqs. (15) and (16). Good agreement is observed in Figure 6, which demonstrates that no calculation of eigenfrequencies is necessary because the particles equilibrate spontaneously on a time scale of $0.1 \mathrm{ps}$, which is much shorter than the total simulation running time, which is about 100 ps.

Figure 7 shows the simulated dependence of the interatomic potential energy and translational order parameter on the temperature during heating and cooling of bulk aluminium in vacuum. During the heating process, between $1,000 \mathrm{~K}$ and $1,300 \mathrm{~K}$, an $\mathrm{S}$ shape is observed in the interatomic potential energy. This characteristic pattern indicates the phase transition from solid to liquid. This result is consistent with those from other studies $[11,16]$. Increase of disorder is noted in the decrease of the translational order parameter between these two 

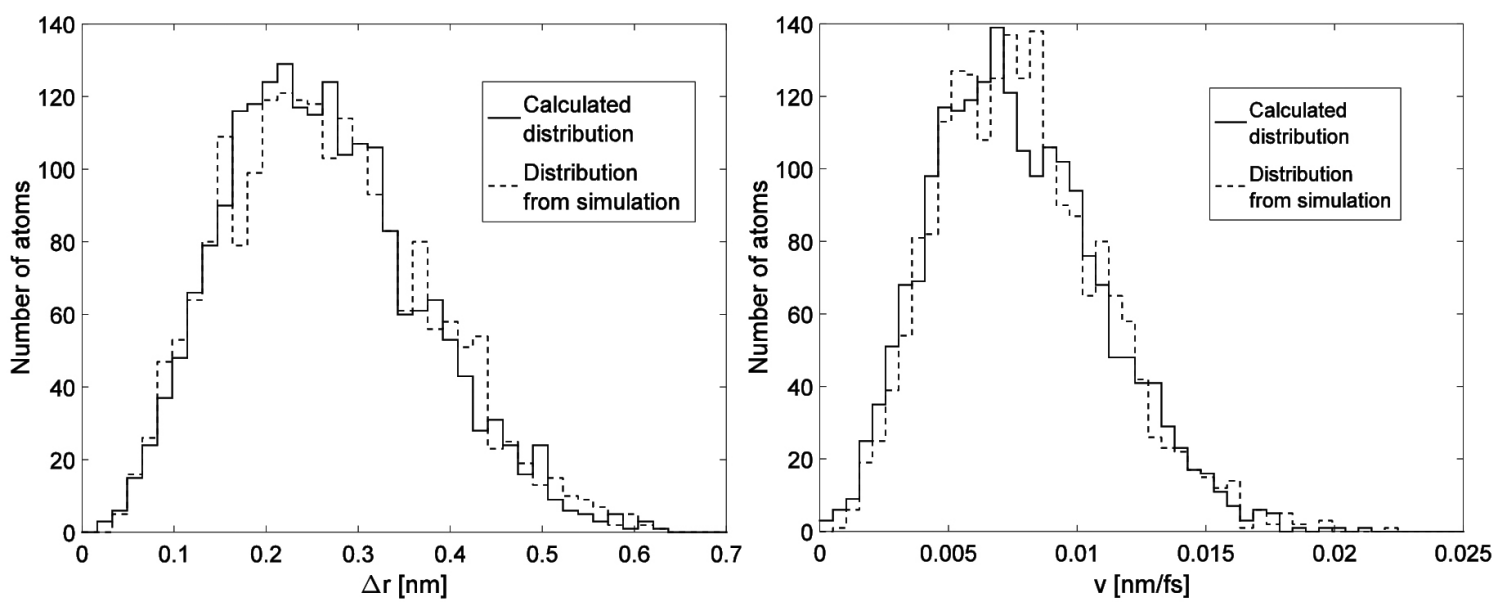

Figure 6: Comparison of calculated position and velocity fluctuations to those obtained from simulation.
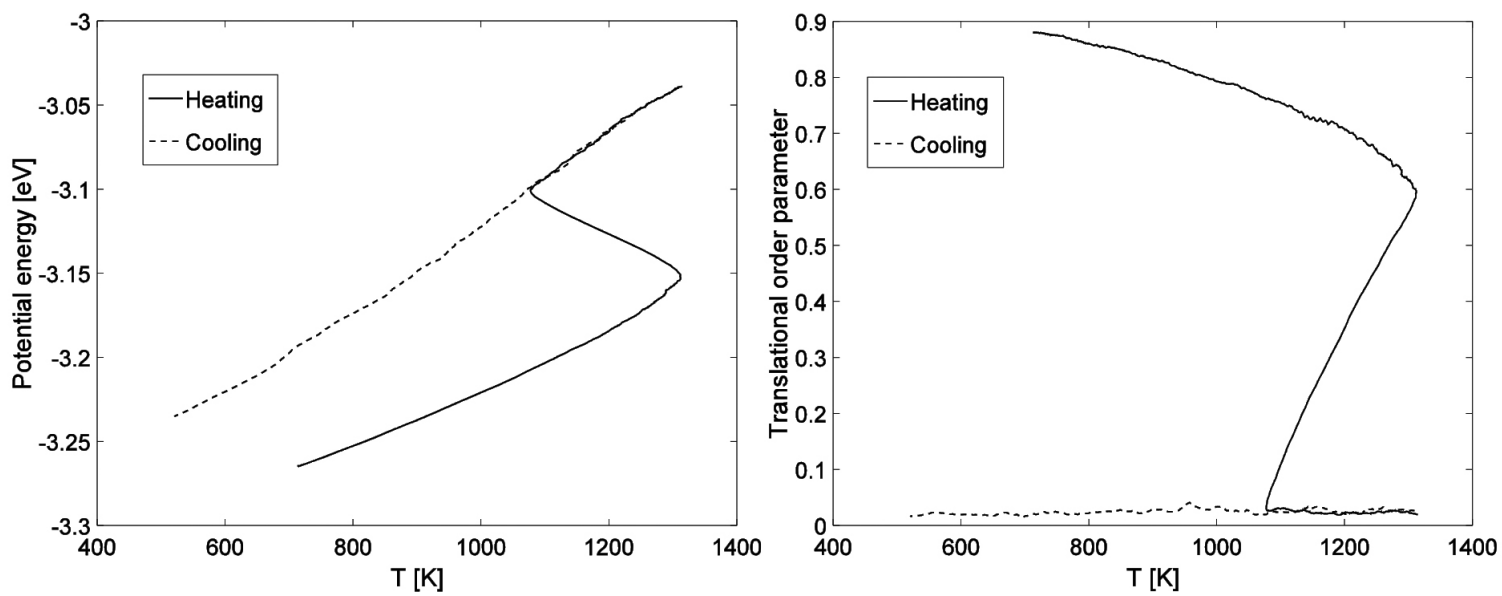

Figure 7: Simulated dependence of potential energy and translational order parameter on temperature during heating and cooling of bulk aluminium sample in vacuum.

temperatures. The temperature during phase change does not remain constant. This can be explained by the absence of point defects, dislocations and grain boundaries, which act as nucleation sites for phase transition, in the solid state of our sample. Their absence results in the production of metastable overheated solid state $[17,18]$. Therefore, the melting point is higher than the one obtained from the experiment. In the experiment, the melting of aluminium is observed around $933.5 \mathrm{~K}$.

In the cooling process, the dependence of potential energy and translational order parameter on temperature does not coincide with the dependencies in the inverse process. The translational order parameter does not return to the value near one, which demonstrates that the structure does not return to the high-ordered form. This does not necessarily mean that the model fails to predict solidification. It can be the consequence of the very high cooling rate $\left(10^{13} \mathrm{~K} / \mathrm{s}\right)$, which pushes the crystallisation process towards very low temperatures or totally removes phase transition and causes the generation of amorphous solid. Figure 8 displays the temperature dependence of factor $\alpha(t)=1 / N \sum_{i=1}^{N}\left(\vec{R}_{i}(t)-\vec{R}_{i}(t=0)\right)^{2}$ during the cooling process. It is seen that at low temperatures, its value becomes constant. This implies that atoms really get stuck at certain positions and that solid is formed. In the study by Solhjoo et al. [19], molecular dynamics simulation was performed using Sutton-Chen potential. At a rate of $10^{12} \mathrm{~K} / \mathrm{s}$, crystallisation was observed at $460 \mathrm{~K}$, and no crystallisation was observed at a rate of $10^{13} \mathrm{~K} / \mathrm{s}$. 


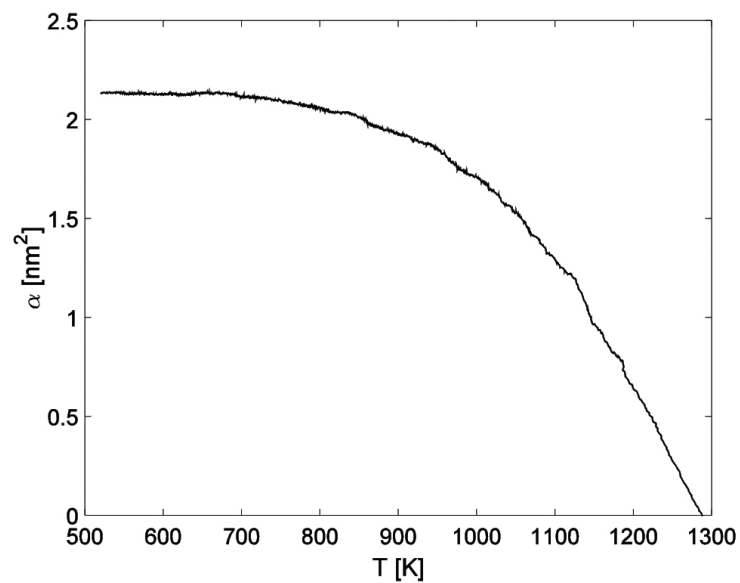

Figure 8: Temperature dependence of factor $\alpha(\mathrm{t})=1 / \mathrm{N} \sum_{\mathrm{i}=1}^{\mathrm{N}}\left(\overrightarrow{\mathrm{R}}_{\mathrm{i}}(\mathrm{t})-\overrightarrow{\mathrm{R}}_{\mathrm{i}}(\mathrm{t}=0)\right)^{2}$ during the cooling process.

\section{Conclusion}

Molecular dynamics simulation using isobaric-isoenthalpic model and glue potential has been performed to simulate the melting of bulk aluminium. A new algorithm for bookkeeping of neighbouring relationships of the atoms has been tested for the first time and used to shorten the computational time of the simulation. Using this algorithm, time consumption can be reduced at least by 5-6 times for a sample with 2,048 atoms. The characteristic equilibration time has also been investigated, which has been found to be 0.1 ps.

Melting occurs between 1,000 K and 1,300 K. Melting temperature does not remain constant during phase transition and is higher than the one provided by the experiment. This is the consequence of the absence of crystal defects. No significant changes in translational order parameter and interatomic potential energy are noted during the cooling process. This can be explained by the very high cooling rate, which causes the formation of amorphous solid.

The next step of this research is the application of point defects, dislocations and grain boundaries. The final goal of research is to investigate the effect of crystal defects on the enthalpy of fusion and on the microscopic short-range order of the melt produced. It is desirable to also observe the solidification process. This will be tried by reduction of the cooling rate to the level that computational resources will approve.
In addition, application of high pressure during the cooling process will also be tested because high pressure leads to a strong tendency of crystallisation [20].

\section{Acknowledgements}

This research was supported by The Faculty of Natural Sciences and Engineering of Ljubljana. The author extends special thanks to Matjaž Berčič for participating in the discussions and for scientific guidance.

\section{References}

[1] Ercolessi, F., Adams, J. B. (1994): Interatomic potentials from first-principles calculations: the force-matching method. Europhysics Letters, 26, pp. 583-588.

[2] Frenkel, D., Smit, B. (2002): Understanding Molecular Simulation. San Diego: Academic Press; pp. 545-558.

[3] Bombač, D., Kugler, G. (2015): Influence of diffusion asymmetry on kinetic pathways in binary $\mathrm{Fe}-\mathrm{Cu}$ alloy: a kinetic Monte Carlo study. Journal of Materials Engineering and Performance, 24, pp. 2382-2389.

[4] Heffelfinger, G. S., Swol, F. (1994): Diffusion in Lennard-Jones fluids using dual control volume grand canonical molecular dynamics simulation (DCV-GCMD). Journal of Chemical Physics, 100, pp. 7548-7552.

[5] Tulley, C. T., Gilmer, G. H. (1979): Molecular dynamics of surface diffusion. I. The motion of adatoms and clusters. Journal of Chemical Physics, 71, pp. 79687972.

[6] Ivanov, V. A., Mishin, Y. (2008): Dynamics of grain boundary motion coupled to shear deformation: an analytical model and its verification by molecular dynamics. Physical Review B, 78, 064106.

[7] Schönfelder, B., Wolf, D., Phillpot, S. R., Furtkamp, M. (1997): Molecular-dynamics method for the simulation of grain-boundary migration. Interface Science, 5 , pp. 245-262.

[8] Yamakov, V., Wolf, D., Phillpot, S. R., Mukherjee, A. K., Gleiter, H. (2002): Dislocation processes in the deformation of nanocrystalline aluminium by molecular-dynamics simulation. Nature Materials, 1, pp. 45-49.

[9] Yamakov, V., Wolf, D., Salazar, M., Phillpot, S. R., Gleiter, H. (2001): Length-scale effects in the nucleation of extended dislocations in nanocrystalline $\mathrm{Al}$ by 
molecular-dynamics simulation. Acta Materialia, 49, pp. 2713-2722.

[10] Alavi, S., Thompson, D. L. (2006): Molecular dynamics simulations of the melting of aluminum nanoparticles. Journal of Physical Chemistry A, 110, pp. 15181523.

[11] Puri, P., Yang, V. (2007): Effect of particle size on melting of aluminum at nano scales. Journal of Physical Chemistry C, 111, pp. 11776-11783.

[12] Andersen, H. C. (1980): Molecular dynamics simulation at constant pressure and/or temperature. Journal of Physical Chemistry, 72, pp. 2384-2393.

[13] Davey, W. P. (1925): Precision measurements of the lattice constants of twelve common metals. Physical Review, 25, pp. 753-761.

[14] Landau, L. D., Lifshitz, E. M. (1980): Statistical Physics. London: Pergamon; $87 \mathrm{p}$.

[15] Allen, M. P., Tildesley, D. J. (1989): Computer Simulation of Liquids. Oxford: Oxford Science; pp. 73-75.
[16] Jin, Z. H., Lu, K. (1998): Melting of surface-free bulk single crystals. Philosophical Magazine Letters, 78, pp. 29-35.

[17] Phillpot, S. R., Lutsko, J. F., Wolf, D., Yip, S. (1989): Molecular-dynamics study of lattice-defect-nucleated melting in silicon. Physical Review B, 40, pp. 28312840.

[18] Mei, Q. S., Lu, K. (2007): Melting and superheating of crystalline solids: from bulk to nanocrystals. Progress in Materials Science, 52, pp. 1175-1262.

[19] Solhjoo, S., Simchi, A., Aashuri, H. (2012): Molecular dynamics simulation of melting, solidification and remelting processes of aluminum. Transactions of $\mathrm{Me}$ chanical Engineering, 36, pp. 13-23.

[20] Sarkar, A., Barat, P., Mukherjee, P. (2006): Molecular dynamics simulation of rapid solidification of Aluminum under pressure. International Journal of Modern Physics B, 22, pp. 2781-2785. 